\title{
Nano-imprinted rear-side diffraction gratings for absorption enhancement in solar cells
}

\author{
Alexander Mellor , Hubert Hauser , Aron Guttowski, Christine Wellens , Benedikt Bläsi , Ignacio \\ Tobías, Antonio Martí, Antonio Luque
}

\begin{abstract}
As wafer-based solar cells become thinner, light-trapping textures for absorption enhancement will gain in importance. In this work, crystalline silicon wafers were textured with wavelength-scale diffraction grating surface textures by nanoimprint lithography using interference lithography as a mastering technology. This technique allows fine-tailored nanostructures to be realized on large areas with high throughput. Solar cell precursors were fabricated, with the surface textures on the rear side, for optical absorption measurements. Large absorption enhancements are observed in the wavelength range in which the silicon wafer absorbs weakly. It is shown experimentally that bi-periodic crossed gratings perform better than uni-periodic linear gratings. Optical simulations have been made of the fabricated structures, allowing the total absorption to be decomposed into useful absorption in the silicon and parasitic absorption in the rear reflector. Using the calculated silicon absorption, promising absorbed photocurrent density enhancements have been calculated for solar cells employing the nano-textures. Finally, first results are presented of a passivation layer deposition technique that planarizes the rear reflector for the purpose of reducing the parasitic absorption.
\end{abstract}

Keywords: solar cell, light trapping, nano-imprint lithography, diffraction grating, crystalline silicon, photonic crystal, absorption enhancement.

\section{INTRODUCTION}

Most commercially produced solar cells use crystalline silicon (c-Si) wafers as light absorbers. Due to the indirect band gap of c-Si, photons with energies close to the band edge are absorbed weakly. By texturing the rear surface of the c-Si wafer, photons are scattered increasing their optical path length in the absorber [1]; a process known as light trapping. This can lead to higher absorption, higher current output, and ultimately higher solar cell efficiencies. Light trapping is expected to gain in importance as the industry moves to thinner wafers in an effort to reduce costs associated to the volume of silicon feedstock used.

One option for a rear-side surface texture is a periodic wavelength-scale diffraction grating etched into the c-Si wafer. This was first proposed for thin-film solar cells by Sheng et al. [2], and was later applied to wafer-based cells by Heine and Morf [3]. Diffraction gratings used for this purpose are typically a few hundred nanometres deep and consume far less of the silicon wafer than conventional surface textures, which have feature sizes of some tens of microns $[4,5]$. What's more, for normal incidence, they offer the possibility to provide greater absorption enhancement than unordered rough surfaces [6].

To be industrially relevant, the diffraction grating must be produced cheaply and quickly. A process chain combining nano-imprint lithography (NIL) with interference lithography as a mastering technology has been developed at the Fraunhofer ISE for this purpose [7,8]. Using this technique, fine-tailored nano-structures can be realized on large areas with high throughput.

In this work, the above mentioned process chain has been used to fabricate wavelength scale linear and crossed diffraction gratings on c-Si wafers. The wafers have been processed into solar cell precursors for optical measurements. Absorption spectra are presented, showing high absorption enhancements in the $1 \mu \mathrm{m}-1.2 \mu \mathrm{m}$ wavelength range in which the c-Si wafer absorbs weakly, and confirming that bi-periodic gratings offer better absorption enhancement than uni-periodic gratings. Optical simulations have been made of the fabricated solar cell precursors. These show excellent 
agreement with the measured spectra, and allow the total absorption to be decomposed into useful absorption in the silicon and parasitic absorption in the reflector. Expected absorbed photocurrent density enhancements are calculated using the results of these simulations. Finally, first results of a method for reducing the parasitic absorption in the rear reflector are presented. This consists in liquid phase deposition of the rear side passivation layer, which leaves a planar surface onto which the reflector can be deposited.

\section{FABRICATION OF REAR TEXTURED SOLAR CELL PRECURSORS BY NANO- IMPRINT LITHOGRAPHY}

Both linear and crossed gratings were fabricated on crystalline silicon substrates using nano-imprint lithography (NIL), with interference lithography as a mastering technology, followed by reactive ion etching (RIE). This technique allows fine-tailored nano-structures to be realized on large areas with high throughput: a necessity for industrialisation. A detailed description of the process chain can be found in Refs. [8,9]. The master structures were realised on photoresist coated glass substrates by two-beam interference lithography followed by a development step. The line grating masters were realised by a single exposure. The crossed grating masters were realised by two exposures with a $90^{\circ}$ rotation of the substrate in between. The inverse pattern of the master structure was replicated on an addition-curing polydimethylsiloxane (PDMS) stamp by cast moulding. The silicon wafer was then coated on the rear side with a low viscosity free-radical curing NIL resist. The stamp was pressed onto the resist and pressure was maintained while the resist was exposed to UV light through the transparent stamp. This left a replica of the master structure on the photoresist. The pattern was then transferred into the underlying silicon wafer by RIE. The RIE was strongly anisotropic, favouring etching normal to the wafer plane. The grating depth is determined by the etching time and can be controlled accurately. The residual photoresist was removed by plasma ashing. SEM micrographs of linear and crossed gratings etched into the silicon substrates are shown in Figure 1 (left) and (right) respectively. It can be seen that the desired binary profile and close to $50 \%$ duty cycle has been achieved in the silicon. A period of $1 \mu \mathrm{m}$ was chosen for the diffraction gratings, this having been found to be optimum for $40 \mu \mathrm{m}$ thick c-Si solar cells in previous numerical studies $[10,11]$. The grating depth was $300 \mathrm{~nm}$ for the line grating and $200 \mathrm{~nm}$ for the crossed grating. These depths had been found to provide the greatest absorption enhancement in preliminary experiments.
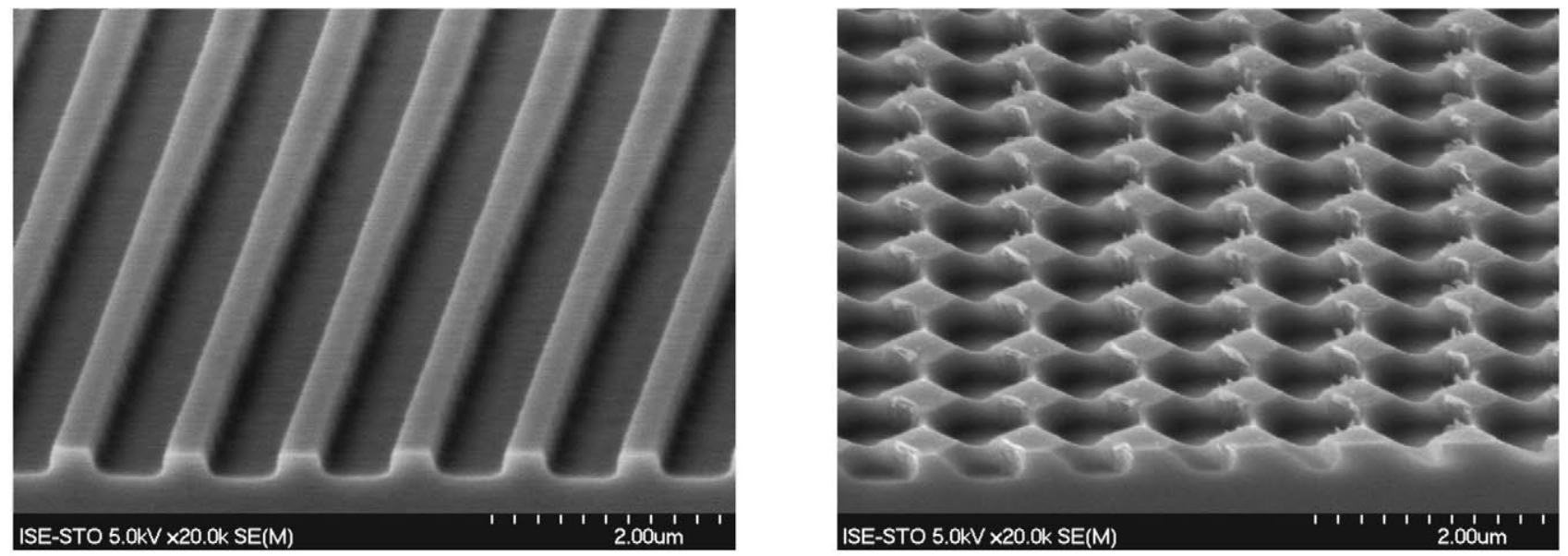

Figure 1. SEM micrographs of Linear (left) and crossed (right) diffraction grating textures in c-Si wafers produced by NIL

Solar cell precursors were fabricated for optical characterisation. Although this work is ultimately aimed at application to wafers with thicknesses around $40 \mu \mathrm{m}$, we have used $200 \mu \mathrm{m}$ thick monocrystalline silicon wafers in this study, due to availability and ease of handling. These were coated with a $63 \mathrm{~nm}$ thick $\mathrm{SiN}$ anti reflection coating (ARC) on the front side. The wafers were textured on the rear side by NIL as described above. $\mathrm{A} \mathrm{SiO}_{2}$ passivation layer was deposited on the textured surface by plasma enhanced chemical vapour deposition (PECVD). Finally, an Al reflector was deposited on the passivation layer by evaporation. An SEM micrograph of the rear-surface cross-section of the linear grating sample is shown in Figure 2. The $\mathrm{SiO}_{2}$ passivation layer has been coloured red for ease of visualization. A planar reference was also fabricated. This consisted of a $200 \mu \mathrm{m}$ thick crystalline silicon wafer with a $63 \mathrm{~nm} \mathrm{SiN} \mathrm{ARC} \mathrm{on} \mathrm{the} \mathrm{front} \mathrm{side} \mathrm{and} \mathrm{a}$ $500 \mathrm{~nm}$ thick $\mathrm{PECVD} \mathrm{SiO} 2$ passivation layer followed by an $\mathrm{Al}$ reflector on the rear side. 


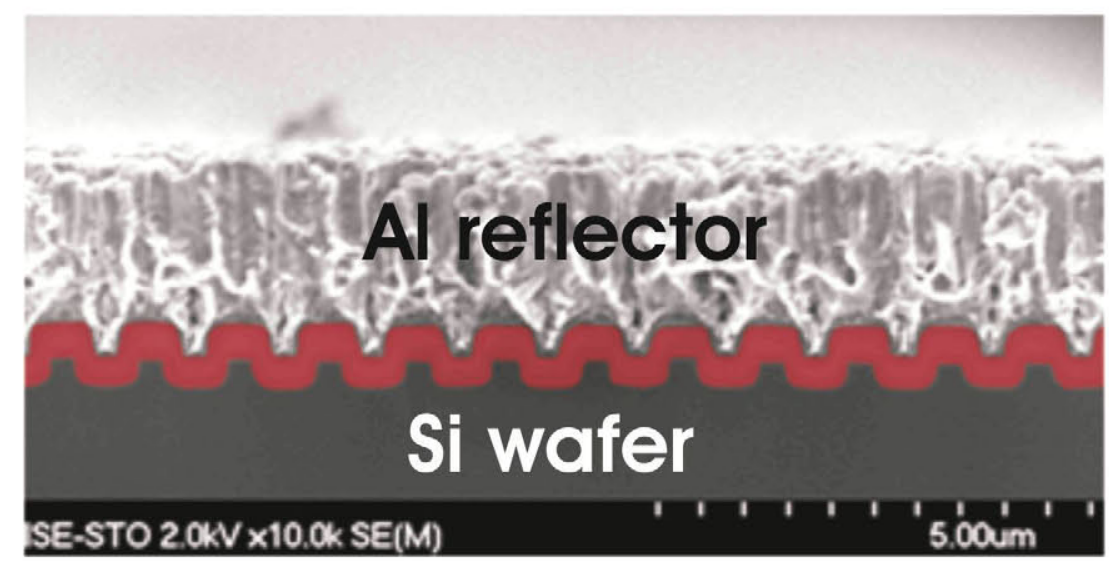

Figure 2. SEM micrograph of the rear-surface cross-section of the linear grating solar cell precursor. The $\mathrm{SiO}_{2}$ passivation layer has been digitally coloured in red for ease of visualization.

\section{MEASURED AND SIMULATED ABSORPTION SPECTRA}

The wavelength dependent absorption of all solar cell precursors was measured by reflection spectroscopy using a Fourier transform spectrometer and an integrating sphere. The absorption was calculated by Absorption = $1-$ Reflection, under the assumption that there is zero transmission through the Al reflector. The absolute absorption enhancement compared to the planar reference is shown for both linear and crossed grating samples in Figure 3. This is the absorption in the textured samples minus the absorption in the planar sample. It can be observed that, in all cases, there is a significant absorption enhancement in the wavelength range close to the silicon band edge in which silicon absorbs weakly $(1 \mu \mathrm{m}-1.2 \mu \mathrm{m})$. The absorption enhancement is much stronger for crossed gratings than for linear gratings. This confirms experimentally the previous theoretical predictions that bi-periodic gratings lead to stronger absorption enhancement than uni-periodic gratings [6, 12].

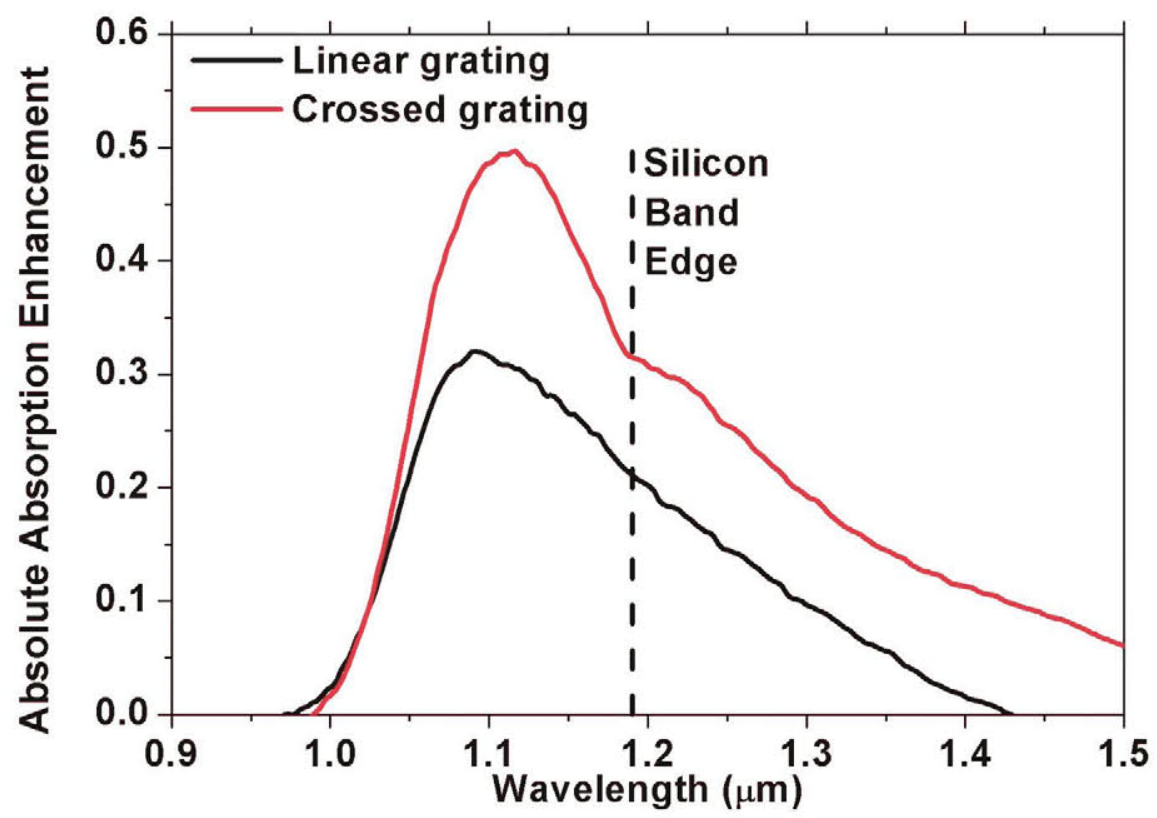

Figure 3. Absolute absorption enhancements for solar cell precursors employing linear and crossed gratings compared to a planar reference. 
The measured absorption is the sum of the useful absorption in the silicon and the parasitic absorption in the reflector. For wavelengths above the silicon band edge $(\approx 1.2 \mu \mathrm{m})$, the silicon is transparent and all absorption takes place in the reflector (it is assumed that the $\mathrm{SiO}_{2}$ is transparent at all wavelengths). The measured absorption enhancement in this range demonstrates that the presence of the grating increases the reflector absorption, as has been predicted in Ref. [11]. To discern how much of the measured absorption occurs in the silicon, and how much occurs in the aluminium reflector, optical simulations have been made of the fabricated structures using the simulation technique presented in Ref. [6].

In the simulation technique, the diffraction-grating region (the textured rear surface of the silicon, the passivation layer and the reflector) is treated separately from the rest of the structure (the c-Si wafer and the ARC). The diffraction-grating region is simulated wave-optically using rigorous coupled wave analysis (RCWA) to yield a far-field intensity scattering matrix. This is combined with a simple steady state matrix formalism, which describes the propagation of the orders within the silicon bulk, their absorption, their partial loss at the front surface, and their re-interaction with the grating via the scattering matrix. The angle dependent reflectivity of the ARC is calculated using a text-book transfer matrix method. Thus the diffraction grating and the ARC are treated coherently, but the bulk absorber is treated incoherently. This is appropriate for structures whose absorber thickness is great than the coherence length of the incident light $\approx 1 \mu \mathrm{m}$ for solar illumination), as is the case for these structures. Because the diffraction grating region and the bulk silicon are simulated separately, it is easy to separate the silicon absorption and the reflector absorption without time consuming near-field spatial interpolation.

Figure 4 shows the measured (red triangles) and simulated (solid black line) total absorption for the solar cell precursors with crossed (a) and linear (b) gratings. Good agreement can be seen between the measured and simulated results, particularly in the case of the linear grating. The simulated absorption has been decomposed into absorption in then silicon wafer and absorption in the aluminium reflector; these are the green and blue curves respectively. It can be seen that, in the light-trapping wavelength range $(1 \mu \mathrm{m}-1.2 \mu \mathrm{m})$, the parasitic reflector absorption is comparable in magnitude to the useful silicon absorption.
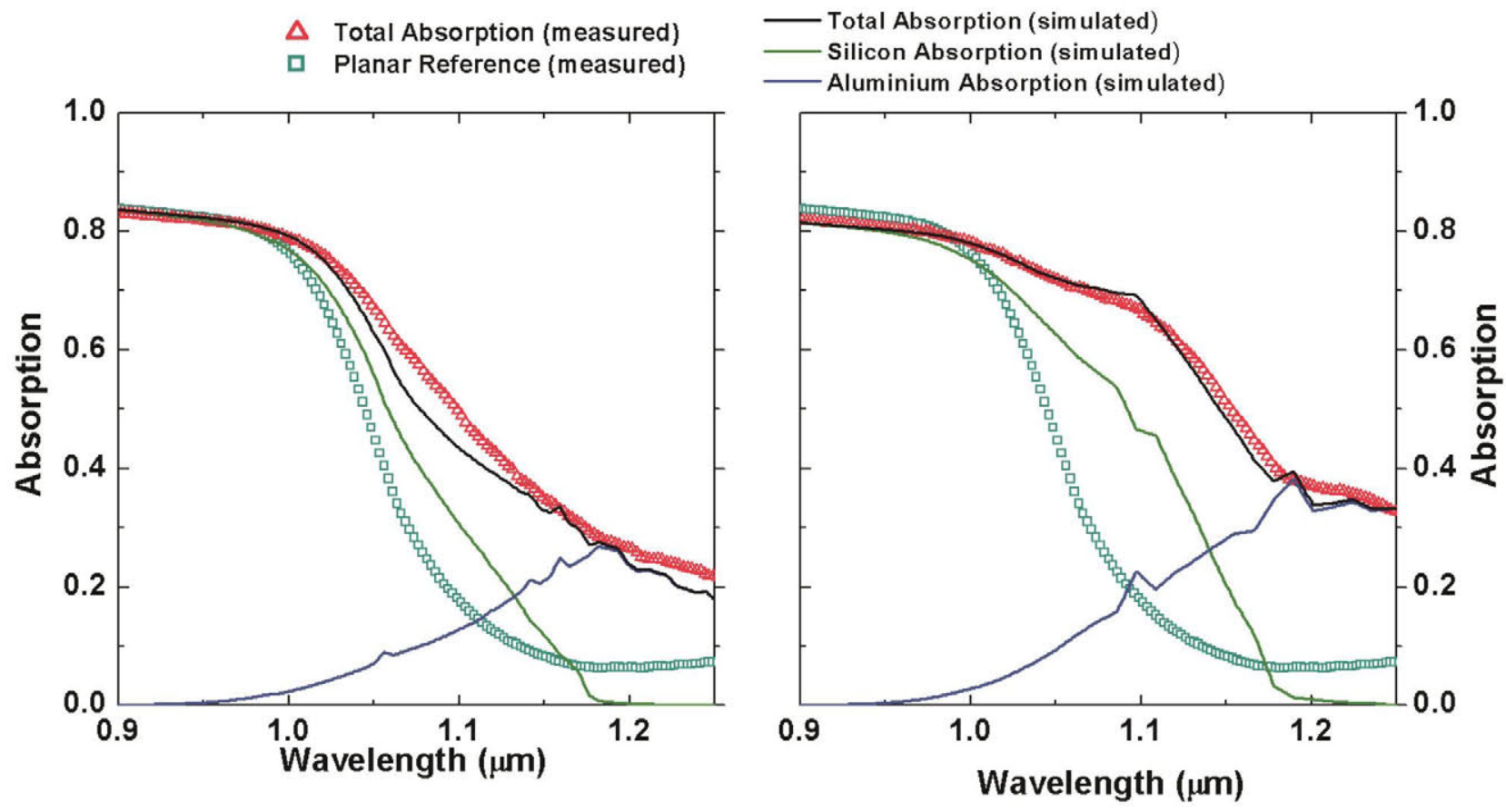

Figure 4. Measured and simulated absorption spectra for the linear (a) and crossed (b) grating solar cell precursors. The simulated total absorption (black curve) is decomposed into the useful silicon absorption (green curve) and the parasitic aluminium absorption (blue curve). Red triangles and blue squares show the measured absorption spectra for the textured sample and the planar reference respectively. 
The absorbed photocurrent density in the silicon $\left(J_{p h, S i}\right)$ and in the aluminium reflector $\left(J_{p h, A l}\right)$ have been calculated by

$$
\begin{aligned}
& J_{p h, S i}=q_{e} \int A_{S i} \Phi_{A m 1.5 G} d \lambda \\
& J_{p h, A l}=q_{e} \int_{\lambda<1.2 \mu m} A_{A l} \Phi_{A m 1.5 G} d \lambda
\end{aligned}
$$

where $\Phi_{A m 1.5 G}$ is photon flux of the AM1.5G spectrum, $q_{e}$ is the elementary charge, is the useful silicon absorption (green curves in Figure 4) and is the absorption in the aluminium reflector (blue curves in Figure 4). The $J_{p h, S i}$ is the current density that would be extracted from the cell if all the electron-hole pairs generated in the silicon reached the external contacts; it represents an upper bound for the short circuit current density $\left(J_{s c}\right)$. The $J_{p h, A l}$ is simply the number of photons absorbed in the aluminium reflector represented as a current density for easy comparison. An upper integration limit of $1.2 \mu \mathrm{m}$ has been chosen so as to only include useful photons that are wastefully absorbed in the reflector.

Table 1 shows the calculated $J_{p h, S i}$ and $J_{p h, A l}$ for solar cells employing the linear and crossed grating textures as well as the planar reference structure, as well as the absolute $J_{p h, S i}$ and $J_{p h, A l}$ enhancement of the textured cells compared to the planar reference, denoted $\Delta J_{p h, S i}$ and $\Delta_{p h, A l}$ respectively and shown in bold.

Table 1. Calculated $J_{p h, S i}$ and $J_{p h, A l}$ for $200 \mu \mathrm{m}$ thick solar cells with a planar rear side and with rear-side linear and crossed grating surface textures. $\Delta$ denotes the absolute difference between the textured solar cell and the planar reference.

\begin{tabular}{r|ccc}
$\begin{array}{c}\text { all units are } \\
\mathrm{mAcm}^{-2}\end{array}$ & $\begin{array}{c}\text { Planar } \\
\text { reference }\end{array}$ & $\begin{array}{c}\text { Linear } \\
\text { grating }\end{array}$ & $\begin{array}{c}\text { Crossed } \\
\text { grating }\end{array}$ \\
\hline$J_{p h, S i}$ & 36.75 & 37.61 & 38.30 \\
$\Delta \boldsymbol{J}_{p h, S i}$ & & $\mathbf{0 . 8 6}$ & $\mathbf{1 . 5 5}$ \\
$J_{p h, A l}$ & 0.19 & 1.06 & 1.34 \\
$\Delta J_{p h, A l}$ & & $\mathbf{0 . 8 7}$ & $\mathbf{1 . 1 5}$
\end{tabular}

These results show that significant $J_{p h, S i}$ enhancements can be expected for solar cells employing rear side diffraction gratings, even for cells as thick as $200 \mu \mathrm{m}$. The expected $J_{p h, S i}$ enhancement for the crossed grating is almost twice that of the linear grating. It can also be seen from Table 1 that the diffraction grating causes a parasitic absorption enhancement that is roughly equal to the useful absorption enhancement. Photons absorbed in this way might otherwise have been absorbed in the silicon and converted to external current. It is therefore expected that by reducing the reflector absorption in this range, greater silicon absorption and therefore greater $J_{p h, S i}$ enhancements than those presented in Table 1 could be achieved. A prospective means of reducing this parasitic absorption is presented in the following section.

All experimental and numerical results presented so far are for $200 \mu \mathrm{m}$ thick wafers. To give an idea of what might be achieved for thinner solar cells, simulations have been made of $40 \mu \mathrm{m}$ thick solar cells employing the grating structures described in Section 2. The $J_{p h, S i}$ and $\Delta J_{p h, S i}$ have been calculated as before and are presented in Table 2. The $J_{p h, S i}$ enhancements are more pronounced for a $40 \mu \mathrm{m}$ cell than for a $200 \mu \mathrm{m}$ cell, as would be expected. The expected $J_{p h, S i}$ for a $40 \mu \mathrm{m}$ solar cell with rear-side crossed grating is similar to that of a $200 \mu \mathrm{m}$ cell with no grating.

Table 1. Calculated $J_{p h, S i}$ for $40 \mu \mathrm{m}$ thick solar cells with a planar rear side and with rear-side linear and crossed grating surface textures.

\begin{tabular}{r|ccc}
$\begin{array}{c}\text { all units are } \\
\mathrm{mAcm}^{-2}\end{array}$ & $\begin{array}{c}\text { Planar } \\
\text { reference }\end{array}$ & $\begin{array}{c}\text { Linear } \\
\text { grating }\end{array}$ & $\begin{array}{c}\text { Crossed } \\
\text { grating }\end{array}$ \\
\hline$J_{p h, S i}$ & 33.89 & 35.17 & 36.52 \\
$\Delta J_{p h, S i}$ & & $\mathbf{1 . 2 8}$ & $\mathbf{2 . 6 3}$
\end{tabular}




\section{PLANARIZATION OF THE REAR REFLECTOR}

Numerical studies of similar structures have shown that the parasitic reflector absorption can be decreased by around $50 \%$ if the aluminium reflector is planar, as opposed to conformal with the Si grating[11]. The reflectors in the structures studied here are clear conformal (Figure 2). This is due to the vapour phase PECVD $\mathrm{SiO}_{2}$ deposition process, which deposits a conformal layer of $\mathrm{SiO}_{2}$ onto the textured silicon surface. In order to deposit a planar reflector, the PECVD process has been replaced by a liquid phase $\mathrm{SiO}_{2}$ deposition. This consists in deposition of a dispersion of colloidal $\mathrm{SiO}_{2}$ nano-particles onto the textured surface by spin coating. This liquid phase process leaves a planar $\mathrm{SiO}_{2}$ surface even when the underlying substrate is textured. This can be seen in Figure 2 (top), which shows a spin-coated $\mathrm{SiO}_{2}$ layer on top of a textured photoresist-on-glass substrate after drying in ambient conditions. Figure 2 (bottom) shows a solar cell precursor with a linear grating, spin-coated passivation layer and $\mathrm{Al}$ reflector. It can be seen that the addition of the $\mathrm{Al}$ reflector causes the previously flat $\mathrm{SiO}_{2}$ surface to modulate slightly, causing a slight modulation of the $\mathrm{Al}$ reflector. We expect that this effect occurs because of the tempering during the evaporation process. Nonetheless, the $\mathrm{Al}$ surface is clearly more planar for the spin-coating method than for the PECVD method. Perfection of this method and investigation into the effect the reflector planarization has on the parasitic reflector absorption and the useful silicon absorption is a subject of on-going research.

\section{$\mathrm{SiO}_{2}$ surface}
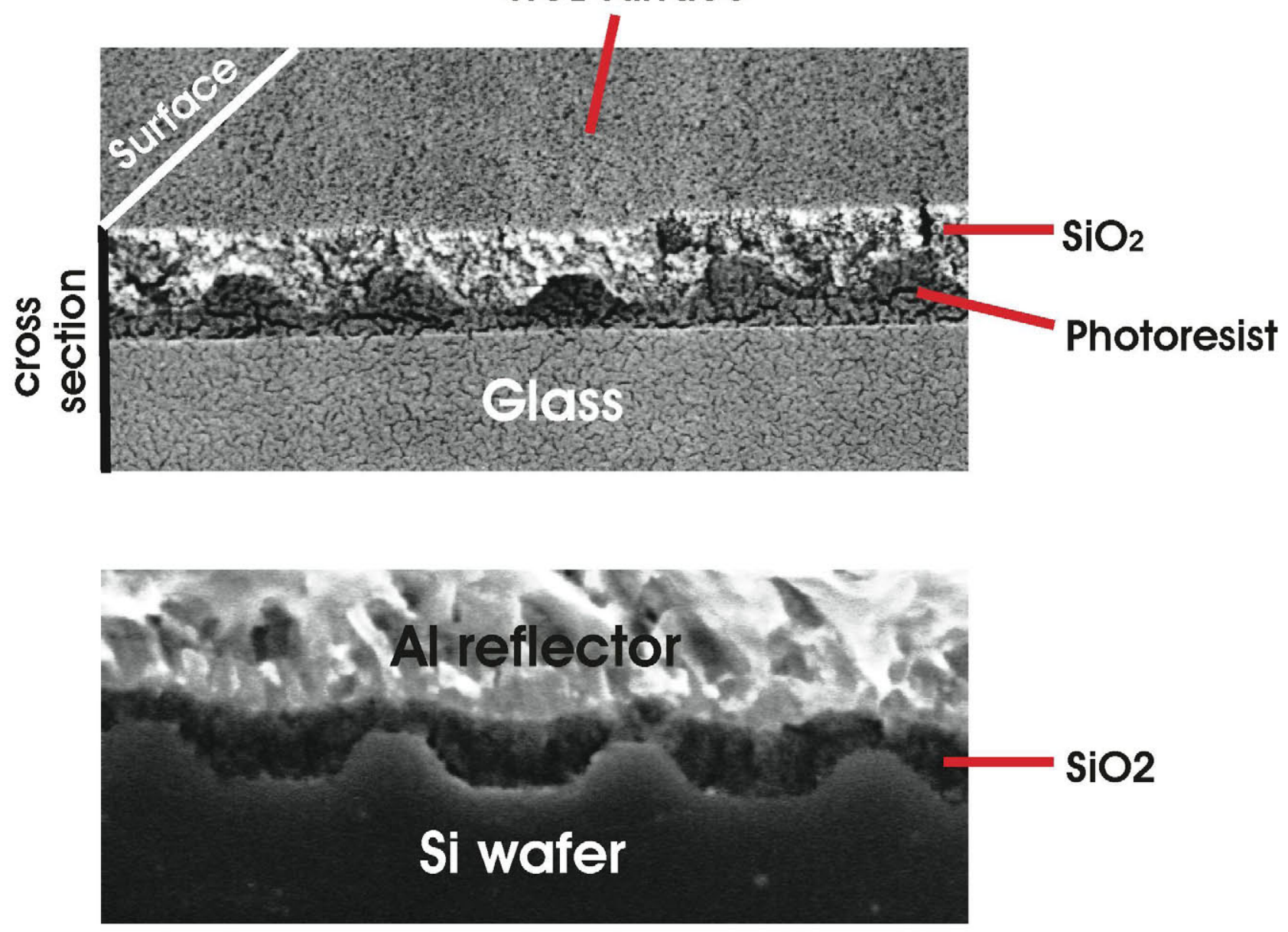

Figure 5. Top: a planar $\mathrm{SiO}_{2}$ layer deposited on a textured photo-resist-on-glass substrate via spin coating. Bottom: cross section of a linear-grating-textured c-Si wafer coated with $\mathrm{SiO}_{2}$ via spin-coating followed by evaporation of an aluminium reflector. Note that the bottom image is upside down with respect to the top image. 


\section{CONCLUSIONS}

c-Si wafers have been textured with wavelength-scale diffraction gratings by NIL using interference lithography as a mastering technology. The wafers have been processed into solar cell precursors for optical characterisation. Measured absorption spectra show a significant absorption enhancement in the wavelength range in which the c-Si wafers absorb weakly $(1 \mu \mathrm{m}-1.2 \mu \mathrm{m})$. Bi-periodic crossed gratings are shown to produce greater absorption enhancements than uniperiodic linear gratings. Optical simulations have been made of the solar cell precursors allowing the total absorption to be decomposed into useful absorption in the silicon and parasitic absorption in the aluminium reflector. The total absorption calculated by the simulations shows good agreement with the measured absorption. Based on the calculated silicon absorption, absorbed photocurrent enhancements have been calculated for solar cells employing the nanotextures. These are $0.86(1.28)$ and $1.55(2.63) \mathrm{mAcm}^{-2}$ for $200 \mu \mathrm{m}(40 \mu \mathrm{m})$ thick c-Si solar cells employing linear and crossed gratings respectively. It has also been shown that the introduction of the grating causes the parasitic reflector absorption to increase by as much as the useful silicon absorption. First results of a method for planarization of the rear reflector to prevent this have been presented.

\section{ACKNOWLEDGEMENTS}

Parts of this work were funded by the Comunidad de Madrid under contract number S2009/ENE-1477 ( Numnacia-II), the Spanish Science Ministry under contract number ENE2009-14481-C02-01(NANOGEFFES), the German Federal Ministry of Environment, Nature Conservation and Nuclear Safety under contract numbers 0325176 (NanoTex) and 0329849A (ThETA), and the German Federal Ministry of Education and Research under contract number 03SF0401 (InfraVolt). Alexander Mellor gratefully acknowledges the Comunidad de Madrid for financial support through the scholarship Personal Investigador de Apoyo.

\section{REFERENCES}

[1] Yablonovitch, E. and Cody, G. D., "Intensity Enhancement in Textured Optical Sheets for Solar Cells," IEEE Trans. Elect. Dev., Ed-29(2), 300 (1982).

[2] Sheng, P. , Bloch, A. N. and Stepleman R. S., "Wavelength-selective absorption enhancement in thin-film solar cells," Applied Physics Letters, 43(6), 579-581 (1983).

[3] Heine, C., and Morf, R. H., "Submicrometer gratings for solar energy applications," Appl. Opt., 34(14), 2476$2482(1995)$.

[4] Zhao, J., Wang, A., Green M. A. et al., "19.8\% efficient "honeycomb" textured multicrystalline and 24.4\% monocrystalline silicon solar cells," Applied Physics Letters, 73(14), 1991-1993 (1998).

[5] Zhao, J., Wang, A., and Green, M. A., "24.5\% Efficiency silicon PERT cells on MCZ substrates and 24.7\% efficiency PERL cells on FZ substrates," Progress in Photovoltaics: Research and Applications, 7(6), 471-474 (1999).

[6] Mellor, A., Tobías, I., Martí, A. et al., "Upper limits to absorption enhancement in thick solar cells using diffraction gratings," Progress in Photovoltaics: Research and Applications, 19(6), 676-687 (2011).

[7] Hauser, H., Michl, B., Kübler, V. et al., "Nanoimprint Lithography for Honeycomb Texturing of Multicrystalline Silicon," Energy Procedia, 8, 648-653 (2011).

[8] Bläsi, B., Hauser, H., Walk, C. et al., [Photon Management Structures Based on Interference Lithography and Nanoimprint Processes], Hamburg(2011).

[9] Bläsi, B., Hauser, H., Höhn O. et al., "Photon Management Structures Originated by Interference Lithography," Energy Procedia, 8, 712-718 (2011).

[10]Mellor, A., Tobías, I., Martí A. et al., "A numerical study of Bi-periodic binary diffraction gratings for solar cell applications," Solar Energy Materials and Solar Cells, 95(12), 3527-3535 (2011).

[11]Peters, M., Rüdiger, M., Hauser H. et al., "Diffractive gratings for crystalline silicon solar cells—optimum parameters and loss mechanisms," Progress in Photovoltaics: Research and Applications, n/a-n/a (2011).

[12] Yu, Z., Raman, A. and Fan, S. "Fundamental limit of nanophotonic light trapping in solar cells," Proceedings of the National Academy of Sciences USA, 107, 17491-17496 (2010). 